\title{
Plaster for Provocation Test Dosage Form
}

National Cancer Institute

\section{Source}

National Cancer Institute. Plaster for Provocation Test Dosage Form. NCI Thesaurus.

Code C149762.

Solid flexible preparation containing an allergen product intended for provocation testing by application to the skin. 